\title{
Increasing the reliability of the soil slopes design using evolutionary modelling
}

\author{
Maxim Prokurov ${ }^{1}$, Alexander Indykin ${ }^{1}$, and Anatoly Alekseytsev ${ }^{2, *}$ \\ ${ }^{1}$ Bryansk State Engineering and Technology University, Stanke Dimitrov av., 3, Bryansk, 241037, \\ Russia \\ ${ }^{2}$ Moscow State University of Civil Engineering, Yaroslavskoe shosse, 26, Moscow, 129337, Russia
}

\begin{abstract}
An issue has been discussed on determining the best value of quadrangular trench slopes, grade considering the provision of reliability for soil steady position in minimizing the quantity of the necessary earthworks. The calculation algorithm has been described including the determination of stability coefficient for a soil slope being in the ultimate rock equilibrium condition. The task is completed via the method of circular-cylindrical revolving surfaces using the genetic algorithm.
\end{abstract}

\section{Introduction}

Designing the slopes for excavations and embankments requires carrying out the correct evaluation of soil position stability. In this case, mistakes in designing may lead to landslide developments in form of displacement of significant earth quantities giving rise to emergency situations occurrence. Natural slopes may also appear unstable that are operated in view of expansion of municipal and industrial areas $[1,6,9,11]$.

Designing the ditches and trenches is carried out taking into account the soil conditions of the construction yard and the foreseeable efficiency of earthworks. Comprehensive consideration of these factors increases the quality of design solutions, which is relevant for the practical implementation of engineering tasks.

It is noted elsewhere that when making calculations and substantiating the soil structure profile while ensuring its stability, it is possible to achieve a reduction in the volume of excavated soil in the arrangement trench slopes up to $25 \%$ [14], in comparison to practical methods that use reference data in determining the dimensions of the said elements.

Nowadays, the designing practices employ various engineering methods of soil structure slope stability evaluation. The studies $[3,8,16]$ conducted has proved, that any known calculation method cannot to high reliability determine the value of the soil structure slope stability coefficient.

To address these challenges, it is proposed to apply the genetic optimization algorithm to the task of the trench slope stability evaluation, which provides a reduction in the amount of earthworks during its arrangement. The studies [2, 4, 5, 10] conducted proved the possibility of effective using the random search methods in solving such a problem.

*Corresponding author: aalexw@mail.ru 


\section{Formulation and optimization problem solution method}

The issue of determining the steepness of slopes of the trenches erected in non-uniform ground arrays is being considered as a typical design problem. Defining the minimum possible length of the slope contributes to a reduction in the overall dimensions of the soil structure in the urban are constraint environment and reduces the amount of the necessary earthworks.

The initial data for designing in this case are: the required dimensions along the bottom of the trench (Fig. 1), trench design depth, the angles of the internal friction of the component soils, adhesion coefficients, soil specific gravity, value of a possible distributed load.

a)

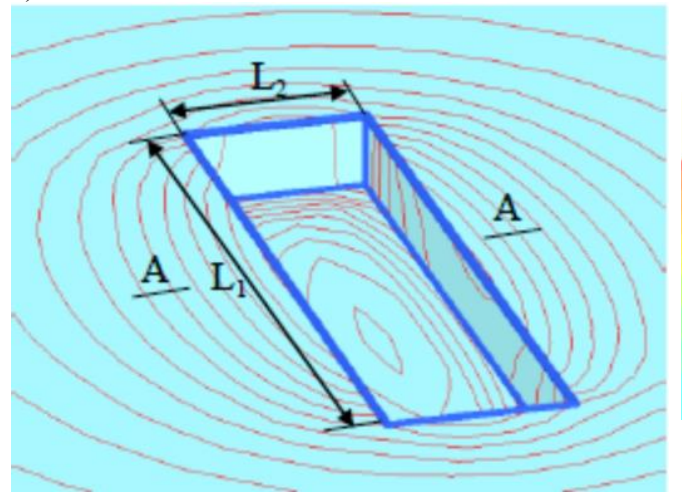

b)

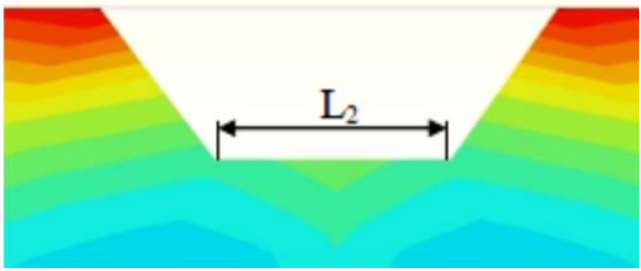

Fig. 1. Design dimensions of a quadrangular ditch: a - ditch sides, assigned by the construction plan; b - ditch cross-section

The algorithm for determining the best value of trench slope grade considering the provision of soil steady position in minimizing the earthworks quantity consists of the following steps:

1) The rational outline of the inclined surface of the soil structure is determined based on the known geometric data - the required trench depth, using the genetic algorithm. The trench outline is given by the following polygonal continuous piecewise linear function [12]:

$$
y(x)=Q\left(x, x_{0}\right) \operatorname{tg} \alpha+y_{b}(x)+Q\left(x, x_{n}\right) \operatorname{tg} \alpha_{n},
$$

where $\alpha$-slope angle to the horizontal surface to $X^{\prime}$ axis $(0<\alpha<\pi / 2)$, function $y_{b}(x)-$ is determined according to polynomial expression (2):

$$
y_{b}(x)=y_{0}+\sum_{k=1}\left(y_{k}-y_{k-1}\right) P\left(x, x_{k-1}, x_{k}-x_{k-1}\right)
$$

The adjustment of the function describing the inclined surface crossfall, is determined by the value of the working volume enlargement factor calculated by the formula:

$$
k_{c}=1+\frac{L_{1}+L_{2}}{L_{1} L_{2}} l+\frac{4}{3 L_{1} L_{2}} l^{2},
$$

where $l$ - trench slope length (determined by the projection of polygon line coordinates to the horizontal), $\mathrm{m}$.

As noted in [6], the function will comply with the best solution in aming the mentioned coefficient for a figure of one. It should be emphasized that when choosing the outline of 
the slope profile, the developed algorithm takes into account the possibility of cohesive soil forming a vertical wall, which maximum height is defined by the formula:

$$
h_{0}=\frac{2 c \cdot \cos \varphi}{\gamma(1-\sin \varphi)},
$$

where $\gamma$ - soil specific weight, $\mathrm{kN} / \mathrm{m} 3 ; \varphi-$ the angle of the internal friction, grade; $c$ - soil adhesion, $\mathrm{kN} / \mathrm{m} 2$.

2) Soil structure slope stability evaluation by the method of circular-cylindrical revolving surfaces. Stability criterion looks in the following way:

$$
k_{s t} \geq 1 \text {. }
$$

The value of stability coefficient $k_{s t}$ is determined as the ratio of moments of retaining and shearing forces related to soil sliding wedge:

$$
k_{s t}=\frac{\sum M_{r e s}}{\sum M_{s h}} .
$$

Stability evaluation task reduces to finding such a soil mass rotation point at which the value of the mentioned coefficient (6) takes the minimum value. In general terms, the criterion of terminating the process of searching for the desired rotation center corresponding to the most dangerous position of the soil mass running surface can be written as follows:

$$
k_{s t}^{\min }<k_{s t(1)}, k_{s t(2)}, k_{s t(3)}, \ldots, k_{s t(i-1)}, k_{s t(i)}, k_{s t(i+1)},
$$

where $k_{s t}^{\min }$ - stability coefficient desired value; $k_{s t(1)}, k_{s t(2)}, k_{s t(3)}, \ldots, k_{s t(i-1)}, k_{s t(i)}, k_{s t(i+1)}-$ intermediate values of this coefficient corresponding to different stages of solution search.

2.1) Searching for sliding wedge rotational center coordinate is carried out according to genetic algorithm [13, 15, 17], having a variety of advantages comparing to other optimization methods. The basic idea of using evolutionary algorithms involves constructing a set (population) of acceptable solutions of the optimization problem and applying random transformations (standard operators of the genetic algorithm) in order to obtain higher quality solutions. Task solution can be written as follows:

$$
k_{s t}=\min \{F(x, y): x \in X ; y \in Y\},
$$

where $X=\{x\}, Y=\{y\}$.

Sliding wedge rotational center position point must be found $O 1$ with coordinates $(x ; y)$, at which function takes minimal value (fig. 2). Sliding wedge rotational center coordinates $(x ; y)$ are given in a form of individuals, consisting of a range of genes presenting concurrent binary recording their current values. Each coordinate pair is assigned the value of stability coefficitend (target function), which will determine the evolution adaptability sign.

The transition from Descartes's space of parameters to the Hemming space of binary strings is accomplished by encoding the variables $x x_{1}, x_{2}, \ldots, x_{N}\left(y_{1}, y_{2}, \ldots, y_{N}\right)$ into binary whole-numbers of a specified length sufficient to provide the desired accuracy in task solution.

2.2) For arbitrarily assigned coordinates of rotation center, slide faces are being constructed with the appropriate radius outlining soil sliding wedge; 


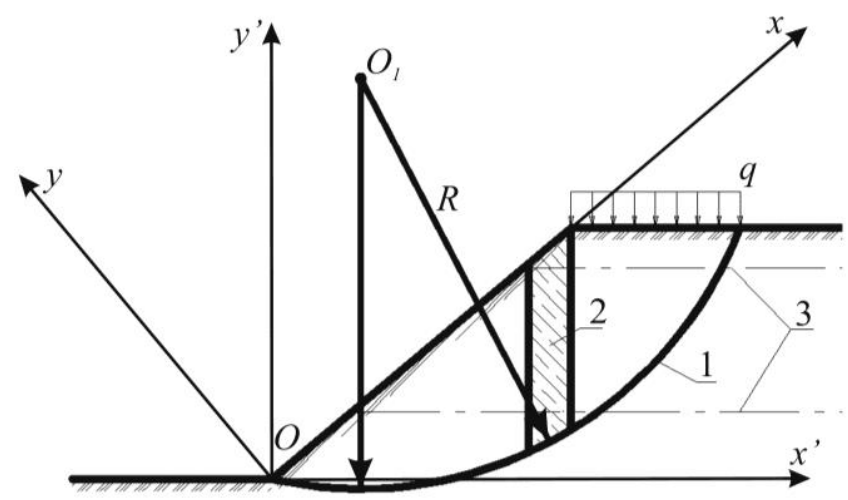

Fig. 2. The area of searching for sliding wedge rotational center position: 1 - slide face outline; $2-$ sliding wedge elementary chamber; 3 - layer bounds of component soil; $x^{\prime} O y^{\prime}-$ coordinate system using ditch profile outlines in selecting; $x O y$-coordinate system using sliding wedges in determining the required parameters

2.3) The sliding wedge is split into a range of chambers (pos. 2, fig. 2) based on the condition of geometrical regularity of their upper and lower outline;

2.4) For each soil chamber, current calculated parameters are determined, provided for in the task assignment;

2.5) Based on the data obtained, moments of retaining and shearing forces are calculated.

2.6) Based on expression (6), soil structure stability coefficient is determined, corresponding to the current rotation center;

2.7) Minimizing stability coefficient coordinates by making random changes to current coordinates of sliding wedge rotation centers, implemented according to simple genetic algorithm procedure.

2.8) If the value of the stability coefficient does not conform the criterion (5), the selected outline of the trench slope profile is considered unacceptable for these soil conditions and requires changes.

3) Verification of the decision based on the limit equilibrium equation of a soil mass.

As noted in [7], in determining the stability coefficient value, one may obtain a range of "false" sliding wedge rotation centers that are not the final task solution as they do not correspond to the limit state of the studied soil mass.

In this respect, it is advisable to check the obtained stability coefficient value for validity. Inspection of compliance of the calculated minimized values of slope stability coefficients with the soil limit equilibrium is performed on the basis of the known expression:

$$
\sum T_{i}-\sum N_{i} \operatorname{tg} \varphi-c L=0,
$$

where $L$ - slide arch length; $T_{i}, N_{i}$ - tangent and normal components of the own weight of $i$ - soil chamber, respectively.

4) Final choice of the shape profile outline, which corresponds to condition (9).

\section{Optimal designing results}

Testing the algorithm for searching the minimum value of the stability coefficient was performed on the basis of a comparison of the obtained value with the calculation results on a number of widely used design methods. A slope was considered having the following 
parameters: length $L=3000 \mathrm{~mm}$; hight $H=2500 \mathrm{~mm} ;-$ my internal friction angle $\varphi=20$ deg.; adhesion coefficient $c=3,5 \mathrm{kPa}$; soil specific weight $\gamma=19 \mathrm{kN} / \mathrm{m} 3$.

The calculation was performed not taking into account the external load and soil nonuniformity, as not all designing methods can consider it.

For calculation, the following characteristics of genetic algorithm were accepted:

- crossover probability; $v 1=40 \%$;

- mutation probability; $v 2=55 \%$;

- inversion probability; $v 3=5 \%$.

Stability coefficient value $\mathrm{k}_{\mathrm{st}}=1,13$ was the result of calculation, calculated relative to the sliding wedge rotational center $O(x ; y)=(410 ; 3250) \mathrm{mm}$ and circle radius $R=3280$ MM. Calculation results, obtained for a number of known design methods are shown in Table 1.

Table 1. Stability coefficient, determined by test methods

\begin{tabular}{|c|l|c|}
\hline $\mathbf{s} / \mathbf{r}$ & \multicolumn{1}{|c|}{ Name of calculation method } & Value of coefficient, $\mathbf{k}_{\mathbf{s t}}$ \\
\hline 1 & $\begin{array}{l}\text { Method of circular-cylindrical sliding surfaces (semi - } \\
\text { graphical way of determining the rotation center) }\end{array}$ & 1.20 \\
\hline 2 & $\begin{array}{l}\text { Method of circular-cylindrical sliding surfaces (using } \\
\text { Yambu graphs) }\end{array}$ & 1.23 \\
\hline 3 & Method by M.H. Goldstein - G.I. Ter-Stepanyan & 1.29 \\
\hline
\end{tabular}

Based on the data in Table 1, it can be assumed that the developed algorithm for calculating the stability coefficient value by the method of circular-cylindrical revolving surfaces using the genetic algorithm of optimization search gives an acceptable accuracy of the problem solution. The calculated value of stability coefficient of the considered ground slope turned out to be somewhat smaller in comparison to solutions obtained in the implementation of the accepted test methods. The implementation of the constructed algorithm is related to the amended exploration of the parameters of the sliding wedge chambers (calculation of the areas of the chambers and the coordinates of their centers of gravity).

\section{Discussion}

The position on the plane sliding surface for the contact of the method is determined by two coordinates, at the same time it is expedient to proceed to determine the center of the collapse prism rotation in the soil space. In addition, in the development of research can be taken into account the structure of the soil. In some cases, this structure is not homogeneous, but is composed of several layers. In this instance, it is necessary to take into account the mutual slip of the layers, which will require the use of very efficient procedures based on genetic algorithms, for example, considered in the studies [2, 17].

\section{Conclusion}

Algorithm for optimal designing the rectangular trench has been developed, which provides the necessary initial reliability of soil slopes and minimizes the amount of earthworks during its arrangement. Searching for the minimum value of soil structure slopes stability coefficient was made using a simple genetic algorithm. Testing the optimization search procedure showed acceptable accuracy in assessing the stability of engineering structure slopes. 


\section{References}

1. M.Y. Prokurov, Indykin A.A. Industrial and Civ. Constr. 10, 75-78 (2015)

2. I.N. Serpik, A.V. Alekseytsev, P.Y. Balabin, Periodica Polytechnika Civil Engineering 61 (3), 471-482 (2017)

3. J. Bojorque, G. De Roeck, J. Maertens, Comp. and Geotechn. 34, 137-150 (2007)

4. I.N. Serpik, A.V. Alekseytsev, Magazine of Civil Engineering 1 (61), 14-24 (2016)

5. Q. Jin-Li, L. Bo, L.I. Yan-Yan J. of China Coal Society 35(9), 1466-1469 (2010)

6. M.Y. Prokurov, Indykin A.A. Construction-2016 (Mat. II Bryansk Int. forum Bryansk, 2016)

7. A.T.C. Goh Civil Engineering and Environmental Systems, 3(17), 181-211 (2000)

8. I.N. Serpik, A.V. Alekseytsev, IOP Conf. Series: Materials Science and Engineering 365, 052003 (2018)

9. M.Y. Prokurov, Indykin A.A. Problems of innovative biosphere-compatible socioeconomic development in the construction, housing and communal and road complexes (Mat. II Bryansk Int. Sci. Conf., Bryansk, 2010)

10. A.V. Alekseytsev, S.A. Akhremenko, Magazine of Civil Engineering 5 (81), 32-42 (2018) (to be published)

11. M.Y. Prokurov, Indykin A.A. Problems of innovative biosphere-compatible socioeconomic development in the construction, housing and communal and road complexes (Mat. III Bryansk Int. Sci. Conf., Bryansk, 2013)

12. P.G. Dolya Analytical methods of geometric modeling (Kharkov: Kharkov National University, 2013)

13. A.V. Alekseytsev, N.S. Kurchenko, Magazine of Civil Engineering 5 (73), 3-13 (2017)

14. M.Y. Prokurov, Indykin A.A. Construction and reconstruction 2, 26-32 (2010)

15. A.V. Alekseytsev, Magazine of Civil Engineering 5 (40), 28-37 (2013)

16. V.D. Raizer, Reliability of Structures: Analysis and Applications (Backbone Publishing Company, 2009)

17. I.N. Serpik, A.V. Alekseytsev, P.Y. Balabin, N.S. Kurchenko, Magazine of Civil Engineering 8 (76), 181-192 (2017) 